\title{
Ion-acoustic rogue waves in multi-ion plasmas
}

\author{
M. Hassan ${ }^{* 1}$, M. H. Rahman ${ }^{1}$, N. A. Chowdhury ${ }^{* * 1}$, A. Mannan ${ }^{1,3}$, and A. A. Mamun ${ }^{1,2}$ \\ ${ }^{1}$ Department of Physics, Jahangirnagar University, Savar, Dhaka-1342, Bangladesh \\ ${ }^{2}$ Wazed Miah Science Research Center, Jahangirnagar University, Savar, Dhaka-1342, Bangladesh \\ ${ }^{3}$ Institut für Mathematik, Martin Luther Universität Halle-Wittenberg, Halle, Germany \\ Email: *hassan206phy@gmail.com, **nurealam1743phy@gmail.com
}

\section{Abstract}

The basic properties of nonlinear ion-acoustic (IA) waves (IAWs), particularly finite amplitude IA rogue waves (IARWs) in a plasma medium (containing pair ions, iso-thermal positrons and non-thermal electrons) are theoretically investigated by deriving the nonlinear Schrödinger equation (NLSE). The criteria for the modulational instability of IAWs, and the basic features of finite amplitude IARWs are identified. The modulationally stable and unstable regions are determined by the sign of the ratio of the 'dispersive coefficient to the nonlinear coefficient of NLSE. The latter is analyzed to obtain the region for the existence of the IARWs, which corresponds to the unstable region. The shape of the profile of the rogue waves depends on the non-thermal parameter $\alpha$ and the ratio of electron temperature to positron temperature. It is found that the increase in the value of the non-thermal parameter enhances both the amplitude and width of IARWs, and that the enhancement of positron (electron) temperature reduces (enhances) the amplitude and width of IARWs. It is worth to mention that our present investigation may be useful for understanding the salient features of IARWs in space (viz., upper region of Titan's atmosphere, cometary comae, and Earth's ionosphere, etc.) and laboratory (viz., plasma processing reactor and neutral beam sources, etc.) plasmas.

Keywords: NLSE, modulational instability, rogue waves.

\section{Introduction}

The signature of pair-ion (PI) plasma (PIP) has been observed by the Cassini spacecraft [1] in spaces, specially, in upper region of Titan's atmosphere, and is also generated by Oohara et al. 2, 3, 4] in laboratory experiments. PI has also identified in cometary comae [5, 6, 7], Earth's iono'sphere [8, 9, 10, 11, 12], plasma processing reactor [15], neutral beam sources [16], and successively has encouraged many researchers to understand the intrinsic contribution of the PI in recognizing the collective properties of the PIP by investigating ion-plasma waves [3], intermediate frequency waves [3], ion-acoustic (IA) solitary waves (IASWs) [5], IA shock waves (IASHWs) [6], IA Gardner solitons (IAGSs) [7], IA double layers (IADLs) [8], and IA rogue waves (IARWs) $[10,11,12,13,14]$, etc.

Non-thermal particles are appeared in a complex system due to the presence of the external force field, and are governed by the Cairns distribution/non-thermal distribution [17, 18, 19], and have also been identified by the Freja [20] and Viking [21] satellites in the magnetosphere and upper ionosphere of the auroral zone. Paul and Bandyopadhyay [22] investigated dust-ion-acoustic solitary waves in a four components plasma medium having non-thermal plasma species, and observed that the height of the positive super-solitons increases with increasing the value of non-thermality of the plasma species. Selim [23] studied IASWs in a three components PIP medium (PIPM) by considering non-thermal electrons, and found that the height and thickness of the compressive solitons increase with increasing the value of electron's non-thermality. Singh and Lakhina [24] considered a three components plasma model having nonthermal electrons, and studied IA super-solitons, and found that the width of the super-solitons increases with an increase in the value of electrons non-thermality.

Electrostatic rogue waves (RWs) are considered to have appeared due to the nonlinear property of the plasma medium as well as the modulational instability (MI) of the carrier waves, and are governed by the nonlinear Schrödinger equation (NLSE), and have also been identified in the plasma physics [10, 11, 12, 25]. El-Tantawy et al. [10] considered a two components plasma model having inertial ions and nonthermal electrons, and studied the mechanism of the formation of IARWs, and also found that behind the critical value of the non-thermal parameter, the nonlinearity and height of the IARWs increase with the non-thermal parameter. Abdelwahed et al. [11] considered a three components PIPM and observed the IARWs in presence of super-thermal electrons, and found that the height of the IARWs decreases with the positive ion number density. Elwakil et al. [12] analyzed the MI of the IAWs in a PIPM having non-thermal electrons. Rahman et al. [25] studied dust-acoustic RWs in a non-thermal dusty plasma medium, and found that the height of the RWs increases with the non-thermality of the plasma species.

Recently, Sabry et al. [5] studied IASWs in a three components PIPM having non-thermal electrons, and found that the presence of negative ion enhances the amplitude but decreases 
the width of the solitary pulse. El-Labany et al. [8] reported IADLs in a three components PIPM in presence of non-thermal electrons. Jannat et al. [6] examined IASHWs in a four components PIPM having inertial PI as well as inertialess electrons and positrons. It is inevitable to investigate the MI of the IAWs and the formation of the IARWs in a four components PIPM by considering inertial double ions and inertialess iso-thermal positrons as well as non-thermal Cairns distributed electrons.

The outline of the paper is as follows: The governing equations describing our plasma model are presented in Section 2. The standard NLSE is derived in Section 3 The MI of the IAWs is examined in Section 4 . The first-order and second-order RWs are discussed in Section 5 Finally, a brief conclusion is provided in Section 6 .

\section{Governing Equations}

We consider a four components plasma model consisting of negative ions, positive ions, electrons, and positrons. Overall, the charge neutrality condition can be written as $Z_{+} n_{+0}+n_{p 0}=$ $Z_{-} n_{-0}+n_{e 0}$; where $n_{+0}, n_{p 0}, n_{-0}$, and $n_{e 0}$ are, respectively, the equilibrium number densities of positive ions, iso-thermal positrons, negative ions, and non-thermal Cairn's distributed electrons, and also $Z_{+}$and $Z_{-}$are, respectively, the charge state of the positive ions and negative ions. Now, the basic set of normalized equations can be written as

$$
\begin{aligned}
& \frac{\partial n_{+}}{\partial t}+\frac{\partial}{\partial x}\left(n_{+} u_{+}\right)=0, \\
& \frac{\partial u_{+}}{\partial t}+u_{+} \frac{\partial u_{+}}{\partial x}=-\frac{\partial \phi}{\partial x}, \\
& \frac{\partial n_{-}}{\partial t}+\frac{\partial}{\partial x}\left(n_{-} u_{-}\right)=0, \\
& \frac{\partial u_{-}}{\partial t}+u_{-} \frac{\partial u_{-}}{\partial x}=\delta_{1} \frac{\partial \phi}{\partial x}, \\
& \frac{\partial^{2} \phi}{\partial x^{2}}=\delta_{2} n_{e}-\delta_{3} n_{p}+\left(1-\delta_{2}+\delta_{3}\right) n_{-}-n_{+},
\end{aligned}
$$

where $n_{+}$and $n_{-}$are the positive and negative ion number density normalized by their equilibrium value $n_{+0}$ and $n_{-0}$, respectively; $u_{-}$and $u_{+}$are the negative and positive ion fluid speed normalized by wave speed $C_{+}=\left(Z_{+} k_{B} T_{e} / m_{+}\right)^{1 / 2}$ (with $T_{e}$ being the temperature non-thermal electron, $m_{+}$being the positive ion mass, and $k_{B}$ being the Boltzmann constant); $\phi$ is the electrostatic wave potential normalized by $k_{B} T_{e} / e$ (with $e$ being the magnitude of single electron charge); the time and space variables are normalized by $\omega_{p+}^{-1}=\left(m_{+} / 4 \pi Z_{+}^{2} e^{2} n_{+0}\right)^{1 / 2}$ and $\lambda_{D+}=\left(k_{B} T_{e} / 4 \pi e^{2} Z_{+} n_{+0}\right)^{1 / 2}$, respectively. Other plasma parameters are defined as $\delta_{1}=Z_{-} m_{+} / Z_{+} m_{-}, \delta_{2}=n_{e 0} / Z_{+} n_{+0}$, and $\delta_{3}=n_{p 0} / Z_{+} n_{+0}$. Now, the expression for electron number density which is obeying non-thermal Cairn's distribution [17] is given by

$$
n_{e}=\left(1-\beta \phi+\beta \phi^{2}\right) \exp (\phi) \text {, }
$$

where $\beta=4 \alpha /(1+3 \alpha)$, with $\alpha$ being the parameter determining the fast particles present in our plasma model. The positron number density which is iso-thermally distributed is given by

$$
n_{p}=\exp \left(-\delta_{4} \phi\right)
$$

where $\delta_{4}=T_{e} / T_{p}$ and $T_{e}>T_{p}$. Now, by substituting Eq. (6) and (7) into Eq. (5), and expanding up to third order of $\phi$, we get

$$
\begin{aligned}
\frac{\partial^{2} \phi}{\partial x^{2}}+n_{+}= & \left(\delta_{2}-\delta_{3}\right)+\left(1-\delta_{2}+\delta_{3}\right) n_{-} \\
& +H_{1} \phi+H_{2} \phi^{2}+H_{3} \phi^{3}+\cdots
\end{aligned}
$$

where

$$
\begin{aligned}
& H_{1}=\delta_{2}(1-\beta)+\delta_{3} \delta_{4}, \\
& H_{2}=\left[\delta_{2}-\delta_{3} \delta_{4}^{2}\right] / 2, \\
& H_{3}=\left[\delta_{2}(1-\beta+6 \beta)+\delta_{3} \delta_{4}^{3}\right] / 6 .
\end{aligned}
$$

We note that the term on the right hand side of the Eq. (8) is the contribution of electron and positron species.

\section{Derivation of the NLSE}

To study the MI of IAWs, we want to derive the NLSE by employing the reductive perturbation method (RPM) and for that case, we can write the stretched coordinates in the form [26, 27]

$$
\begin{aligned}
& \xi=\epsilon\left(x-v_{g} t\right), \\
& \tau=\epsilon^{2} t,
\end{aligned}
$$

where $v_{g}$ is the group velocity and $\epsilon(\epsilon \ll 1)$ is a small parameter. Then, we can write the dependent variables as [26, 27]

$$
\begin{aligned}
& n_{+}=1+\sum_{m=1}^{\infty} \epsilon^{m} \sum_{l=-\infty}^{\infty} n_{+l}^{(m)}(\xi, \tau) \exp [i l(k x-\omega t)], \\
& u_{+}=\sum_{m=1}^{\infty} \epsilon^{m} \sum_{l=-\infty}^{\infty} u_{+l}^{(m)}(\xi, \tau) \exp [i l(k x-\omega t)], \\
& n_{-}=1+\sum_{m=1}^{\infty} \epsilon^{m} \sum_{l=-\infty}^{\infty} n_{-l}^{(m)}(\xi, \tau) \exp [i l(k x-\omega t)], \\
& u_{-}=\sum_{m=1}^{\infty} \epsilon^{m} \sum_{l=-\infty}^{\infty} u_{-l}^{(m)}(\xi, \tau) \exp [i l(k x-\omega t)], \\
& \phi=\sum_{m=1}^{\infty} \epsilon^{m} \sum_{l=-\infty}^{\infty} \phi_{l}^{(m)}(\xi, \tau) \exp [i l(k x-\omega t)],
\end{aligned}
$$

where $k(\omega)$ is real variables representing the carrier wave number (frequency). The derivative operators in above equations are treated as follows:

$$
\begin{aligned}
& \frac{\partial}{\partial t} \rightarrow \frac{\partial}{\partial t}-\epsilon v_{g} \frac{\partial}{\partial \xi}+\epsilon^{2} \frac{\partial}{\partial \tau}, \\
& \frac{\partial}{\partial x} \rightarrow \frac{\partial}{\partial x}+\epsilon \frac{\partial}{\partial \xi} .
\end{aligned}
$$

Now, by substituting Eq. (9)-(17) into Eq. (1)-(4) and Eq. (8), and collecting the terms containing $\epsilon$, the first order $(m=1$ with 


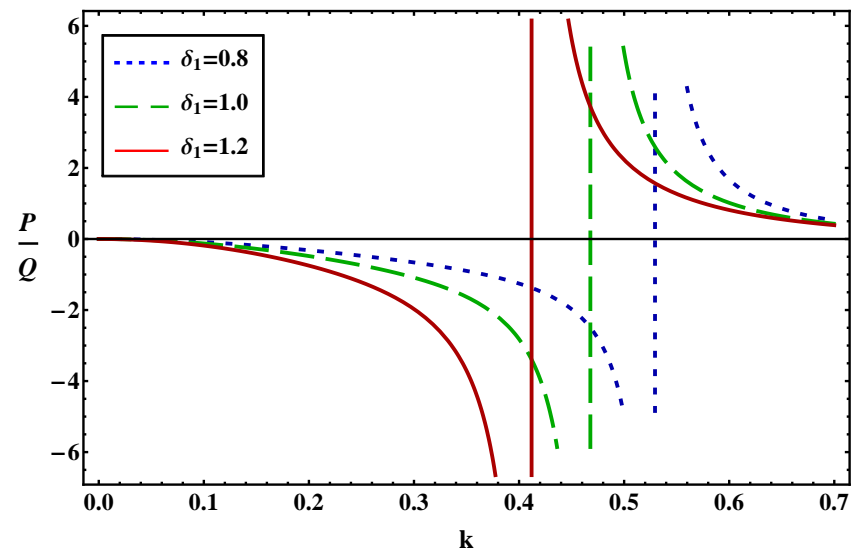

Figure 1: Plot of $P / Q$ vs $k$ for different values of $\delta_{1}$ when other parameters are $\alpha=0.7, \delta_{2}=0.4, \delta_{3}=0.4$, and $\delta_{4}=2.0$.

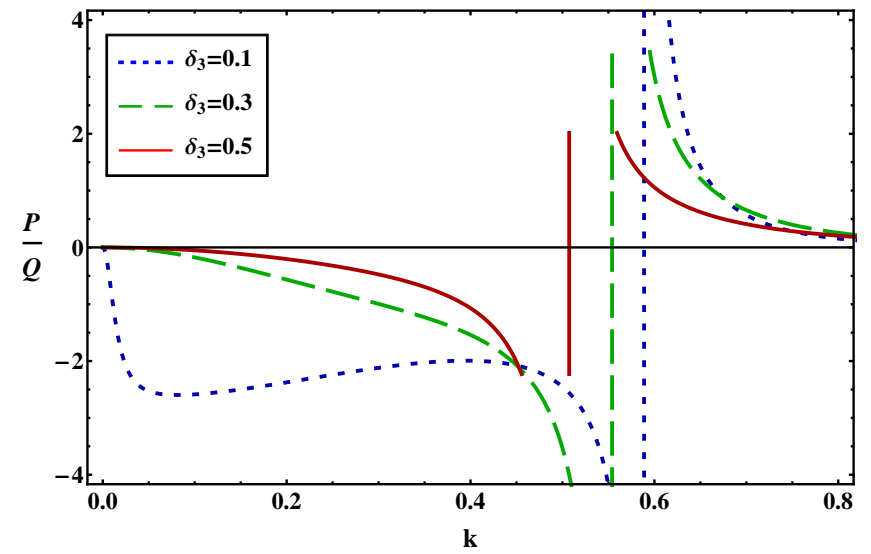

Figure 2: Plot of $P / Q$ vs $k$ for different values of $\delta_{3}$ when other parameters are $\alpha=0.7, \delta_{1}=0.8, \delta_{2}=0.4$, and $\delta_{4}=2.0$.

$l=1)$ reduced equations can be written as

$n_{+1}^{(1)}=\frac{k^{2}}{\omega^{2}} \phi_{1}^{(1)}$,

$u_{+1}^{(1)}=\frac{k}{\omega} \phi_{1}^{(1)}$,

$n_{-1}^{(1)}=-\frac{k^{2} \delta_{1}}{\omega^{2}} \phi_{1}^{(1)}$

$u_{-1}^{(1)}=-\frac{k \delta_{1}}{\omega} \phi_{1}^{(1)}$.

These relation provides the dispersion relation for IAWs

$\omega^{2}=\frac{k^{2}\left[1+\delta_{1}\left(1-\delta_{2}+\delta_{3}\right)\right]}{k^{2}+H_{1}}$.

The second order ( $m=2$ with $l=1$ ) equations are given by

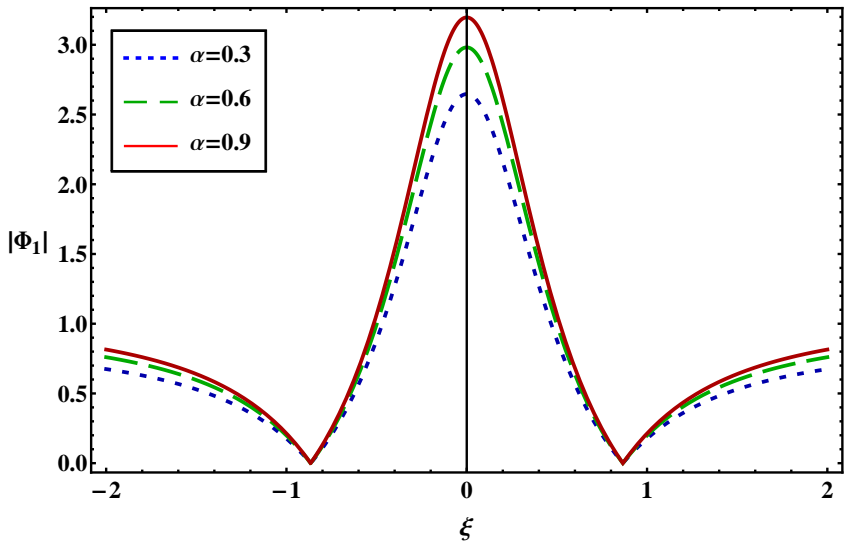

Figure 3: Plot of $\left|\Phi_{1}\right|$ vs $\xi$ for different values of $\alpha$ when other parameters are $k=0.7, \tau=0, \delta_{1}=0.8, \delta_{2}=0.4, \delta_{3}=0.4$, and $\delta_{4}=2.0$.

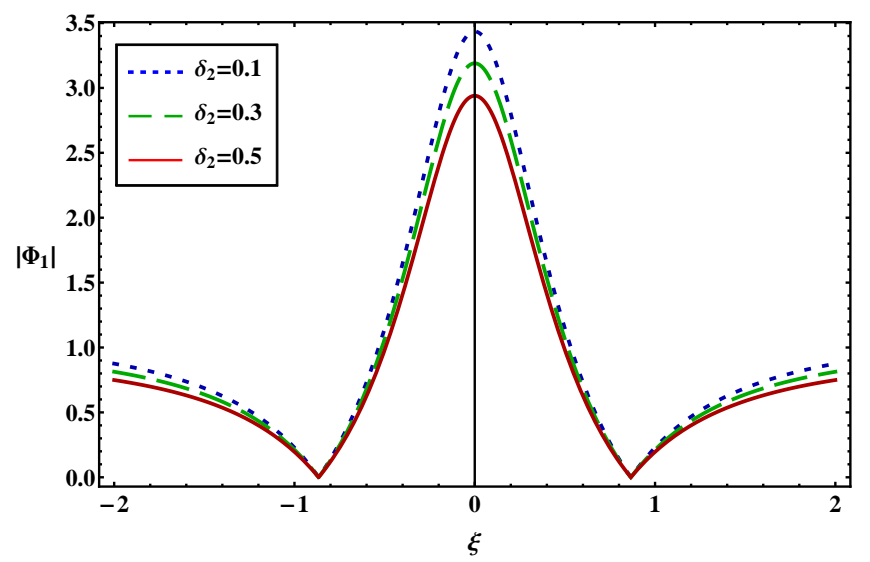

Figure 4: Plot of $\left|\Phi_{1}\right|$ vs $\xi$ for different values of $\delta_{2}$ when other parameters are $k=0.7, \tau=0, \alpha=0.7, \delta_{1}=0.8, \delta_{3}=0.4$, and $\delta_{4}=2.0$.

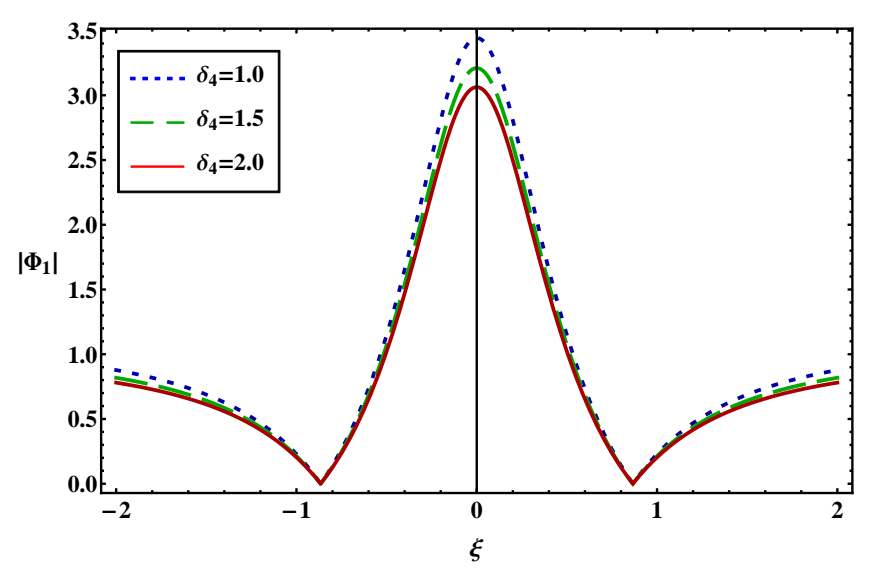

Figure 5: Plot of $\left|\Phi_{1}\right|$ vs $\xi$ for different values of $\delta_{4}$ when other parameters are $k=0.7, \tau=0, \alpha=0.7, \delta_{1}=0.8, \delta_{2}=0.4$, and $\delta_{3}=0.4$. 
$n_{+1}^{(1)}=\frac{k^{2}}{\omega^{2}} \phi_{1}^{(2)}+\frac{2 i k\left(v_{g} k-\omega\right)}{\omega^{3}} \frac{\partial \phi_{1}^{(1)}}{\partial \xi}$,

$u_{+1}^{(2)}=\frac{k}{\omega} \phi_{1}^{(2)}+\frac{i\left(v_{g} k-\omega\right)}{\omega^{2}} \frac{\partial \phi_{1}^{(1)}}{\partial \xi}$,

$n_{-1}^{(2)}=-\frac{\delta_{1} k^{2}}{\omega^{2}} \phi_{1}^{(2)}-\frac{2 i k \delta_{1}\left(v_{g} k-\omega\right)}{\omega^{3}} \frac{\partial \phi_{1}^{(1)}}{\partial \xi}$

$u_{-1}^{(2)}=-\frac{\delta_{1} k}{\omega} \phi_{1}^{(2)}-\frac{i \delta_{1}\left(v_{g} k-\omega\right)}{\omega^{2}} \frac{\partial \phi_{1}^{(1)}}{\partial \xi}$,

with the compatibility condition

$v_{g}=\frac{\omega\left[1+\delta_{1}\left(1-\delta_{2}+\delta_{3}\right)-\omega^{2}\right]}{k\left[1+\delta_{1}\left(1-\delta_{2}+\delta_{3}\right)\right]}$.

The coefficients of $\epsilon$ for $m=2$ and $l=2$ provide the second order harmonic amplitudes which are found to be proportional to $\left|\phi_{1}^{(1)}\right|^{2}$

$n_{+2}^{(2)}=H_{4}\left|\phi_{1}^{(1)}\right|^{2}$,

$u_{+2}^{(2)}=H_{5}\left|\phi_{1}^{(1)}\right|^{2}$,

$n_{-2}^{(2)}=H_{6}\left|\phi_{1}^{(1)}\right|^{2}$,

$u_{-2}^{(2)}=H_{7}\left|\phi_{1}^{(1)}\right|^{2}$,

$\phi_{2}^{(2)}=H_{8}\left|\phi_{1}^{(1)}\right|^{2}$,

where

$$
\begin{aligned}
& H_{4}=\frac{3 k^{4}+2 H_{8} \omega^{2} k^{2}}{2 \omega^{4}}, \\
& H_{5}=\frac{k^{3}+2 \omega^{2} k H_{8}}{2 \omega^{3}}, \\
& H_{6}=\frac{3 \delta_{1}^{2} k^{4}-2 \delta_{1} H_{8} \omega^{2} k^{2}}{2 \omega^{4}}, \\
& H_{7}=\frac{\delta_{1}^{2} k^{3}-2 \omega^{2} k \delta_{1} H_{8}}{2 \omega^{3}}, \\
& H_{8}=\frac{2 H_{2} \omega^{4}+3 \delta_{1} k^{4}\left(1-\delta_{2}+\delta_{3}\right)-3 k^{4}}{2 \omega^{2} k^{2}-2 \omega^{4}\left(4 k^{2}+H_{1}\right)+2 \delta_{1} \omega^{2} k^{2}\left(1-\delta_{2}+\delta_{3}\right)} .
\end{aligned}
$$

Now, we consider the expression for ( $m=3$ with $l=0$ ) and ( $m=2$ with $l=0$ ), which leads zeroth harmonic modes. Thus, we obtain

$$
\begin{aligned}
& n_{+0}^{(2)}=H_{9}\left|\phi_{1}^{(1)}\right|^{2}, \\
& u_{+0}^{(2)}=H_{10}\left|\phi_{1}^{(1)}\right|^{2}, \\
& n_{-0}^{(2)}=H_{11}\left|\phi_{1}^{(1)}\right|^{2}, \\
& u_{-0}^{(2)}=H_{12}\left|\phi_{1}^{(1)}\right|^{2}, \\
& \phi_{0}^{(2)}=H_{13}\left|\phi_{1}^{(1)}\right|^{2},
\end{aligned}
$$

where

$$
\begin{aligned}
& H_{9}=\frac{2 v_{g} k^{3}+\omega k^{2}+\omega^{3} H_{13}}{v_{g}^{2} \omega^{3}}, \\
& H_{10}=\frac{k^{2}+\omega^{2} H_{13}}{v_{g} \omega^{2}}, \\
& H_{11}=\frac{2 v_{g} \delta_{1}^{2} k^{3}+\omega \delta_{1}^{2} k^{2}-\delta_{1} \omega^{3} H_{13}}{v_{g}^{2} \omega^{3}}, \\
& H_{12}=\frac{\delta_{1}^{2} k^{2}-\delta_{1} \omega^{2} H_{13}}{v_{g} \omega^{2}}, \\
& H_{13}=\frac{2 H_{2} v_{g}^{2} \omega^{3}+2 v_{g} \delta_{1}^{2} k^{3}\left(1-\delta_{2}+\delta_{3}\right)+F 1}{\omega^{3}\left[1+\delta_{1}\left(1-\delta_{2}+\delta_{3}\right)-H_{1} v_{g}^{2}\right]},
\end{aligned}
$$

where $F 1=\omega k^{2} \delta_{1}^{2}\left(1-\delta_{2}+\delta_{3}\right)-2 v_{g} k^{3}-\omega k^{2}$. Finally, the third harmonic modes $(m=3)$ and $(l=1)$ and with the help of Eq. (18)-(40), give a set of equations, which can be reduced to the following NLSE:

$$
i \frac{\partial \Phi}{\partial \tau}+P \frac{\partial^{2} \Phi}{\partial \xi^{2}}+Q|\Phi|^{2} \Phi=0
$$

where $\Phi=\phi_{1}^{(1)}$ for simplicity. In Eq. (38), $P$ is the dispersion coefficient which can be written as

$$
P=\frac{3 v_{g}\left(v_{g} k-\omega\right)}{2 \omega k},
$$

and also $Q$ is the nonlinear coefficient which can be written as

$$
\begin{aligned}
Q= & \frac{\omega^{3}}{2 k^{2}\left[1+\delta_{1}\left(1-\delta_{2}+\delta_{2}\right)\right]} \times\left[-\frac{2 k^{3}\left(H_{5}+H_{10}\right)}{\omega^{3}}\right. \\
& +2 H_{2}\left(H_{8}+H_{13}\right)+3 H_{3}-\frac{k^{2}\left(H_{4}+H_{9}\right)}{\omega^{2}} \\
& -\frac{\delta_{1} k^{2}\left(1-\delta_{2}+\delta_{2}\right)\left(H_{6}+H_{11}\right)}{\omega^{2}} \\
& \left.-\frac{2 \delta_{1} k^{3}\left(1-\delta_{2}+\delta_{2}\right)\left(H_{7}+H_{12}\right)}{\omega^{3}}\right] .
\end{aligned}
$$

It may be noted here that both $P$ and $Q$ are function of various plasma parameters such as $\alpha, \delta_{1}, \delta_{2}, \delta_{3}, \delta_{4}$ and $k$. So, all the plasma parameters are used to maintain the nonlinearity and the dispersion properties of the PIPM.

\section{Modulational instability}

The stable and unstable regions of the IAWs are organized by the sign of the dispersion $(P)$ and nonlinear $(Q)$ coefficients of the standard NLSE (30). The stability of IAWs in four components PIPM is governed by the sign of $P$ and $Q$ [26, 27, 28, 29, 30, 31, 32, 33, 34, 35, 36]. When $P$ and $Q$ have same sign (i.e., $P / Q>0$ ), the evolution of the IAWs amplitude is modulationally unstable in presence of the external perturbations. On the other hand, when $P$ and $Q$ have opposite sign (i.e., $P / Q<0$ ), the IAWs are modulationally stable. The plot of $P / Q$ against $k$ yields stable and unstable regions for the IAWs. The point, at which transition of $P / Q$ curve intersects 
with $k$-axis, is known as threshold or critical wave number $k$ $\left(=k_{c}\right)[26,27,28,32,33,34,35,36]$.

We have numerically analyzed the behaviour of $P / Q$ with respect to $k$ for different values of $\delta_{1}$ and $\delta_{3}$ in Figs. 1 and 2 , respectively. It can be seen from Fig. 1 that (a) the modulationally stable region corresponding to the criteria (i.e., $P / Q<0$ ) as well as modulationally unstable region corresponding to the criteria (i.e., $P / Q>0$ ) can be found from this figure; (b) the $k_{c}$ decreases with an increase in the value of $\delta_{1}$; (c) the mass of the negative ions causes to increase the value of $k_{c}$ while the mass of the positive ions causes to decrease the value of $k_{c}$ for a constant value of negative and positive ions charge state (via $\delta_{1}$ ). Figure 2 demonstrates the presence of the Maxwellian inertialess positrons reduces the $k_{c}$ as well as allows to generate IARWs associated with IAWs within the condition $P / Q>0$ in a PIPM for a small value of $k$ when the number density as well as the charge state of the positive ion remain constant (via $\delta_{3}$ ). On the other hand, modulationally stable region (i.e., $P / Q<0$ ) as well as $k_{c}$ enhances with increasing the value of number density and charge state of the positive ion for a constant value of positron population.

\section{Rogue waves}

The NLSE (38) has a variety of solutions, among them there is a hierarchy of rational solution that are localized in both the $\xi$ and $\tau$ variables. Each solution of the hierarchy represents a unique event in space and time, as it increases its amplitude quickly along each variable, reaching its maximum value and finally decays, just as quickly as it appeared [37]. Thus, these waves were nicknamed "waves that appear from nowhere and disappear without a trace [38]". The first-order rational solution of NLSE (38) is given as [32, 33, 34, 35, 36, 37, 38, 39, 40, 41]

$$
\Phi_{1}(\xi, \tau)=\sqrt{\frac{2 P}{Q}}\left[\frac{4+16 i \tau P}{1+4 \xi^{2}+16 \tau^{2} P^{2}}-1\right] \exp (2 i \tau P) .
$$

The nonlinear superposition of the two or more first-order RWs gives rise higher-order RWs and form a higher amplitude more complicated nonlinear structure. The second-order rational solution is expressed as $32,33,34,35,36,37,38,39,40,41,39$, 38]

$$
\Phi_{2}(\xi, \tau)=\sqrt{\frac{P}{Q}}\left[1+\frac{G_{2}(\xi, \tau)+i M_{2}(\xi, \tau)}{D_{2}(\xi, \tau)}\right] \exp (i \tau P),
$$

where

$$
\begin{aligned}
G_{2}(\xi, \tau) & =\frac{3}{8}-6(P \xi \tau)^{2}-10(P \tau)^{4}-\frac{3 \xi^{2}}{2}-9(P \tau)^{2}-\frac{\xi^{4}}{2} \\
M_{2}(\xi, \tau) & =-P \tau\left[\xi^{4}+4(P \xi \tau)^{2}+4(P \tau)^{4}-3 \xi^{2}+2(P \tau)^{2}-\frac{15}{4}\right] \\
D_{2}(\xi, \tau) & =\frac{\xi^{6}}{12}+\frac{\xi^{4}(P \tau)^{2}}{2}+\xi^{2}(P \tau)^{4}+\frac{2(P \tau)^{6}}{3}+\frac{\xi^{4}}{8} \\
& +\frac{9(P \tau)^{4}}{2}-\frac{3(P \xi \tau)^{2}}{2}+\frac{9 \xi^{2}}{16}+\frac{33(P \tau)^{2}}{8}+\frac{3}{32}
\end{aligned}
$$

The solutions (39) and 40 represent the profile of the firstorder and second-order RWs within the modulationally unstable region, which concentrate a significant amount of energy

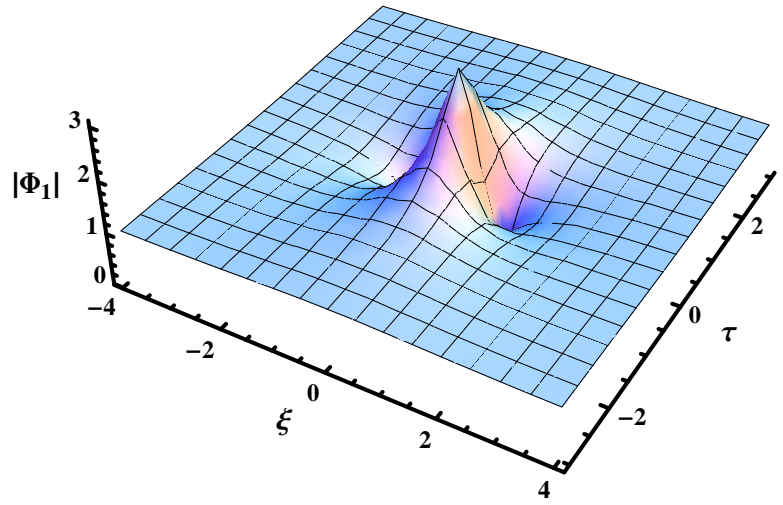

(a)

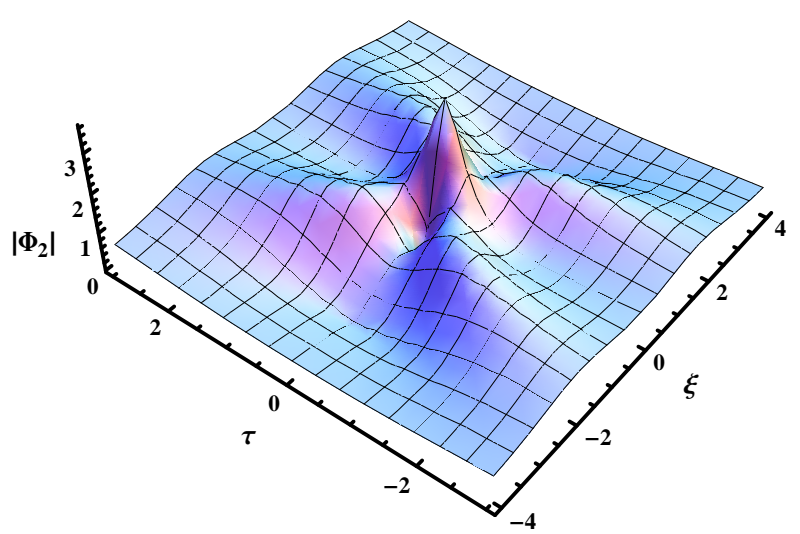

(b)

Figure 6: Profile of the (a) first-order rational solution and (b) second-order rational solution.

into a relatively small area. The properties of the first-order rational solution [by using Eq. (39)] of NLSE (38) in a PIPM can be observed in Figs. 3,5 corresponding different values of $\alpha, \delta_{2}$, and $\delta_{4}$. Figure 3 shows the structure of the first-order IARWs according to the value of electrons non-thermality $(\alpha)$. The increase in the value of $\alpha$ does not only cause to increase the height of the first-order IARWs associated IAWs in the modulationally unstable region (i.e., $P / Q>0$ ) but also causes to increase the thickness of the first-order IARWs associated IAWs in the modulationally unstable region (i.e., $P / Q>0$ ).

The effects of the ratio of the number density of electron to positive ion as well as the charge state of the positive ion (via $\delta_{2}$ ) can be seen from Fig. 4 and it is obvious from this figure that (a) the increase in the value of $\delta_{4}$ causes to decrease the height of the first-order IARWs in the modulationally unstable region (i.e., $P / Q>0$ ) as well as causes to decrease the thickness of the first-order IARWs in the modulationally unstable region (i.e., $P / Q>0$ ); (b) physically, the presence of the excess number 


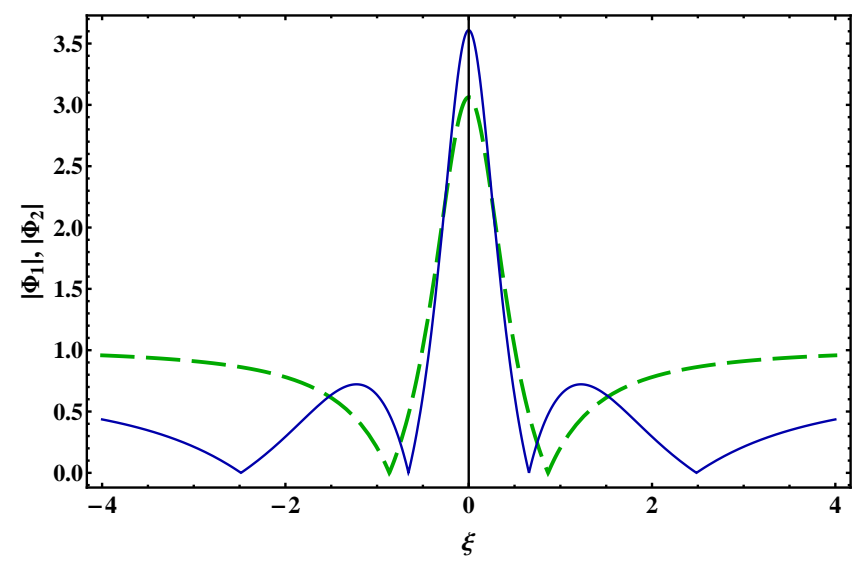

Figure 7: Plot of first-order (dashed green curve) and second-order (solid blue curve) rational solutions of NLSE [38] at $\tau=0$.

of electrons reduces the nonlinearity of the plasma medium as well as the height and thickness of the first-order IARWs while the presence of the excess number of positive ions increases the nonlinearity of the PIPM as well as the height and thickness of the first-order IARWs when the charge state of the positive ion remains constant (via $\delta_{2}$ ).

The nature of the first-order IARWs with the variation of $\delta_{4}$ can be observed from Fig. 5 which clearly indicates that (a) the height and thickness of the first-order IARWs associated with IAWs in the modulationally unstable region (i.e., $P / Q>0$ ) decreases with increasing the value of $\delta_{4}$; (b) the temperature of the positron in the modulationally unstable region of IAWs enhances the nonlinearity of the plasma medium as well as the height and thickness of the first-order IARWs while the temperature of the electron in the modulationally unstable region of IAWs reduces the nonlinearity of the plasma medium as well as the height and thickness of the first-order IARWs.

The space and time evolution of the first-order and secondorder rational solutions of the NLSE (38) can be observed from Figs. 6(a) and 6(b), respectively. Figure 7 indicates the firstorder and second-order solution at $\tau=0$, and it is clear form this figure that (a) the second-order rational solution has double structures compared with first-order rational solution; (b) the height of the second-order rational solution is always greater than the height of the first-order rational solution; (c) the potential profile of the second-order rational solution becomes more spiky (i.e., the taller height and narrower width) than the firstorder rational solution; (d) the second (first) order rational solution has four (two) zeros symmetrically located on the $\xi$-axis; (e) the second (first) order rational solution has three (one) local maxima.

The existence of highly energetic rogue waves has already been confirmed experimentally [42, 43, 44, 45] and theoretically [46]. The second-order rational solution was experimentally observed by Chabchoub et al. [43] in a "Water Wave Tank", and the experimental result regarding the amplification is a nice agreement with the theoretical result. Bailung et al. [44] demonstrated an experiment in a multi-component plasma medium to observe RWs, and found a slowly amplitude modu- lated perturbation undergoes self modulation and gives rise to a high amplitude localized pulse. Rogue waves also observed in fiber optics [45].

\section{Conclusion}

The amplitude modulation of IAWs has been theoretically investigated in an unmagnetized four components PIPM consisting of inertial positively and negatively charged ions as well as inertialess non-thermal electrons and iso-thermal positrons. A NLSE, which governs the MI of IAWs and the formation of electrostatic first-order and second-order RWs in PIPM, is derived by using the RPM. To conclude, we hope that our results may be useful in understanding the nonlinear phenomena (viz., MI of IAWs and IARWs, etc.) in space PIPM (viz., cometary comae [5, 6, 7] and Earth's ionosphere [8, 9, 10, 11, 12]) and laboratory PIPM (viz., plasma processing reactor [15] and neutral beam sources [16], etc.).

\section{Acknowledgements}

M Hassan is thankful to the Bangladesh Ministry of Science and Technology for awarding the National Science and Technology (NST) Fellowship. A Mannan thanks the Alexander von Humboldt Foundation for a Postdoctoral Fellowship.

\section{References}

[1] A. J. Coates, F. J. Crary, G. R. Lewis, D. T. Young, J. H. Waite, and E. C. Sittler, Geophys. Res. Lett. 34 (2007) 22103.

[2] W. Oohara and R. Hatakeyama, Phys. Rev. Lett. 91 (2003) 205005.

[3] W. Oohara, D. Date, and R. Hatakeyama, Phys. Rev. Lett. 95 (2005) 175003.

[4] W. Oohara, Y. Kuwabara, and R. Hatakeyama, Phys. Rev. E 75 (2007) 056403.

[5] R. Sabry, W. M. Moslem, and P. K. Shukla, Phys. Plasmas 16 (2009) 032302 .

[6] N. Jannat, M. Ferdousi, and A. A. Mamun, Commun. Theor. Phys. 64 (2015) 479.

[7] N. Jannat, M. Ferdousi, and A. A. Mamun, Plasma Phys. Rep. 42 (2016) 678.

[8] S. K. El-Labany, R. Sabry, W. F. El-Taibany, and E. A. Elghmaz Astrophys. Space Sci. 340 (2012) 77.

[9] H. Massey, Negative Ions, 3rd ed., Cambridge University Press, Cambridge (1976).

[10] S. A. El-Tantawy, N. A. El-Bedwehy, and S. K. El-Labany, Phys. Plasmas 20 (2013) 072101.

[11] H. G. Abdelwahed, E. K. El-Shewy, M. A. Zahran, and S. A. Elwakil, Phys. Plasmas 23 (2016) 022102.

[12] S. A. Elwakil, E. K. El-Shewy, and H. G. Abdelwahed, Phys. Plasmas 17 (2010) 052301.

[13] N. A. Chowdhury, A. Mannan, M. M. Hasan, and A. A. Mamun, Chaos 27 (2017) 093105.

[14] N. Ahmed, A. Mannan, N. A. Chowdhury, and A. A. Mamun, Chaos 28 (2018) 123107.

[15] R. A. Gottscho, C. E. Gaebe, IEEE Trans. Plasma Sci. 14 (1986) 92.

[16] M. Bacal, G. W. Hamilton, Phys. Rev. Lett. 42 (1979) 1538.

[17] R. A. Cairns, A. A. Mamun, R. Bingham, R. Boström, R. O. Dendy, C. M. C. Nairn, and P. K. Shukla, Geophys. Res. Lett. 22 (1995) 2709.

[18] M. H. Rahman, A. Mannan, N. A. Chowdhury, and A. A. Mamun, Phys. Plasmas 25 (2018) 102118.

[19] N. A. Chowdhury, A. Mannan, M. R. Hossen, and A. A. Mamun, Contrib. Plasma Phys. 58 (2018) 870. 
[20] P. O. Dovner, A. I. Eriksson, R. Boström, and B. Holback, Geophys. Res. Lett. 21 (1994) 1827.

[21] R. Boström, G. Gustafsson, B. Holback, G. Holmgren, H. Koskinen, and P. Kintner, Phys. Rev. Lett. 61 (1988) 82.

[22] A. Paul and A. Bandyopadhyay, Astrophys. Space Sci. 361 (2016) 172.

[23] M. M. Selim, Eur. Phys. J. Plus 131 (2016) 93.

[24] S. V. Singh and G. S. Lakhina, Commun. Nonlinear. Sci. Numer. Simulat. 23 (2015) 274.

[25] M. H. Rahman, N. A. Chowdhury, A. Mannan, M. Rahman, and A. A. Mamun, Chin. J. Phys. 56 (2018) 2061.

[26] I. Kourakis and P. K. Shukla, Phys. Plasmas 10 (2003) 3459.

[27] I. Kourakis and P. K. Shukla, Nonlinear Proc. Geophys. 12 (2003) 407.

[28] R. Fedele, Phys. Scr. 65 (2002) 502.

[29] N. A. Chowdhury, A. Mannan, and A. A. Mamun, Phys. plasmas 24 (2017) 113701

[30] S. Jahan, N. A. Chowdhury, A. Mannan, and A. A. Mamun, Commun. Theor. Phys. 71 (2019) 327

[31] N. A. Chowdhury, A. Mannan, M. M. Hasan, and A. A. Mamun, Vacuum 147 (2018) 31.

[32] S. Guo, L. Mei, A. Sun, Ann. Phys. 332 (2013) 38.

[33] S. Guo, L. Mei, and W. Shi, Phys. Lett. A 377 (2013) 2118.

[34] S. Guo, L. Mei, Phys. Plasmas 21 (2014) 112303.

[35] S. K. El-Labany, W. F. El-Taibany, and N. A. Zedan, Phys. Plasmas 22 (2015) 073702.

[36] S. A. El-Tantawy, N. A. El-Bedwehy, and S. K. El-Labany, Phys. Plasmas 20 (2019) 072102.

[37] A. Ankiewicz, P. A. Clarkson, and N. Akhmediev, J. Phys. A 43 (2010) 12002.

[38] A. Ankiewicz, N. Devine, and N. Akhmediev, Phys. Lett. A 373 (2009) 3997.

[39] N. Akhmediev, A. Ankiewicz, and J. M. Soto-Crespo, Phys. Rev. E 80 (2009) 026601.

[40] N. Akhmediev, A. Ankiewicz, and M. Taki, Phys. Lett. A 373 (2009) 675.

[41] Z. Yan, Phys. Lett. A 374 (2010) 672.

[42] A. Chabchoub, N. P. Hoffmann, and N. Akhmediev, Phys. Rev. Lett. 106 (2011) 204502.

[43] A. Chabchoub, N. Hoffmann, M. Onorato, and N. Akhmediev, Phys. Rev. X 2(2012) 011015 .

[44] H. Bailung, S. K. Sharma, and Y. Nakamura, Phys. Rev. Lett. 107 (2011) 255005.

[45] B. Kibler, J. Fatome, C. Finot, G. Millot, F. Dias, G. Genty, N. Akhmediev, and J. M. Dudley, Nat. Phys. 6 (2010) 790.

[46] Shalini, N. S. Saini, and A. P. Misra, Phys. Plasmas 22 (2015) 092124. 